\title{
A Clinical Investigation about Horizontal Heterophoria Among Newly Diagnosed Myopia Juveniles in Nanchong District of China
}

\section{Ying Li Yan}

North Sichuan Medical College

Long Li

Boshi Eye hospital

Ying Wang

North Sichuan Medical College

Peng Guan Zeng

North Sichuan Medical College

Chun Yun Zou ( $\nabla$ zychun03@163.com )

North Sichuan Medical College https://orcid.org/0000-0003-4905-1231

\section{Research Article}

Keywords: Heterophoria, myopia, juvenile school students

Posted Date: January 31st, 2019

DOI: https://doi.org/10.21203/rs.2.272/v1

License: (9) This work is licensed under a Creative Commons Attribution 4.0 International License. Read Full License 


\section{Abstract}

Background: To establish the prevalence and distribution of heterophoria in low myopia juveniles and and provide evidence of myopia prevention and treatment.

Methods: Near and distant heterophoria were administered to the patients who were diagnosed of low myopia at their first visit in clinic. Tthe Von Graefe method was performed to test near, distant horizontal heterophoria types and degrees. Statistical analysis was performed with SPSS17.0 software.

Results: A total of 150 students were participated in this study. Distant and near phoria was detected in $92.67 \%$ and $96.00 \%$ of the students respectively. exophoria was more common than esophoria(diantant:79.33\% vs $13.33 \%$; near: $86.67 \%$ vs $9.33 \%, P<0.05$ ), and the mean deviation of diantant and near heterophoria was $-1.97 \triangle$ and $-3.91 \triangle$. There was a statistical difference between the near and distant heterophoria $(P<0.05)$.

Conclusions: Most myopia juveniles have different extents of exophoria. Due to the high prevalence of heterophoria in myopia, in the process of refraction, attention should be paid to the examination of binocular visual function parameters such as heterophoria, which is conducive to the issuance of suitable optical correction prescriptions and the reasonable measures for myopia prevention and control.

Keywords: Heterophoria, myopia, juvenile school students

\section{Background}

Heterophoria is the relative deviation of the eyes in absence of fusional vergence. and its examination,both diatance and near, is essential to diagnose binocular disorders [1]. In 2015, Babinsky et al[2] conducted heterophroia exams on 50 children (refractive diopter: $-1.25 \mathrm{D}$ to $+3.75 \mathrm{D}$ ) and 12 orthophoria adults, their results showed that $78 \%$ children and $69 \%$ adults manifested exophoria defined by over two prism degree, and only $20 \%$ children and $23 \%$ adults had orthophoria(the degree of deviation was within two prism degree, including each two-prism-degree exophoria and esophoria), and only one kid and one adult had esphoria over two prism degrees. Meanwhile, some studies suggested the association between esphoria and myopia developments [3], as well as the relationship between esphoria and myopia. Myopia is one of the most common refractive error in juveniles. As applications of progressive eyeglass and attempts of multiple approaches for myopia control keep increasing, scholars pay more and more attention to binocular visual parameters of heterophoria.

The uncoordinated accommodation and convergence in myopia could affect the balance of extraocular muscles, resulting in heterophoria. An abnormal value of heterophoria may lead to symptoms like visual fatigue, headache or double vision[4]. Eyeglass wearing discomforts due to heterophoria is common, especially in those juveniles with convergence insufficiency and higher heterophoria degree. Therefore, measurements of heterophoria will affect further prescriptions. Considering the present high incidence of myopia in juveniles, appropriable optic prescriptions could not only protect their vision but also prevent 
heterophoria and improve their visual functions. However, some optometrists only conduct the degree of refractive errors, especially in the undeveloped area of China, not mentioning binocular vision tests. Therefore, in this study, we performed near and distant heterophoria test on newly diagnosed myopia juveniles and conducted a relevant literature review to evaluate the current situation of imbalanced extraocular muscles. It is expected to provide clinical data for optic prescription and control of myopia.

\section{Methods}

\section{Inclusion criteria}

The juveniles had corrected visual acuity(VA) above 20/20, with fusion and stereovision. None of them had been prescribed any optical correction at the time of their visit. Written informed consent was obtained from the parents of the children tested. Organic diseases were excluded by slit lamp and fundus examination. The school children should be low myopia with or without low astigmatism and low anisometropia (all $<1.00 \mathrm{D}$ ). (spherical degree: from $-0.50 \mathrm{D}$ to $-3.00 \mathrm{D}$, cylindrical degree $<1.00 \mathrm{D}$ ).

\section{Heterophoria examination Procedures}

All the subjects were examined by the same optometrist, with the same instruments under the same conditions. The tests and measurements were as follows: history and symptoms, visual acuity without correction, Objective refraction which was performed by static retinoscopy, monocular subjective refraction by phoropter, followed by binocular balancing to a standard endpoint of maximum plus for best visual acuity (MPBVA). Then Von Graefe method was applied to measure near and distance heterophoria ( $6 \mathrm{~m}$ for distance and $40 \mathrm{~cm}$ for near test). A dissociating prism of $6 \triangle$ base up was placed in front of the right eye and a measuring prism (12 $\triangle$ base in) before the left eye, using the phoropter. For both distance and near vision, subjects were instructed to fixate at the line of $20 / 30$ visual acuity letters and were told to keep the letters clear all the time. All tests for the horizontal heterophoria were done three times and recorded and the average value was analyzed.

\section{Statistical analysis}

The numerical data was expressed as $x(-) \pm S D$, and statistical analysis was conducted with SPSS (version 17.0). Repetitive measurement and analysis method was utilized to compare the near and distance heterphoria. A $P$ value less than 0.05 was treated as statistical significance.

\section{Informed consent and ethical approval of procedures}

The local administration of the Education and School Board were contacted to request their cooperation. After securing permission to perform the study, approval was obtained by the appropriate university Ethical Advisory Committee. Written informed consent from the participants or their parents/legal guardians were obtained before the examination. 


\section{Results}

There were 73 male patients and 77 female juveniles, with ages between 12 to 16 years(mean age: $13.71 \pm 1.25$ years). Their spherical diopter ranged between $-0.50 \mathrm{D}$ to $-3.00 \mathrm{D}$, and astigmatism ranged between $-0.25 \mathrm{D}$ to $-0.75 \mathrm{D}$. Table 1 shows the descriptive statistics for the 150 mild myopia subjects. The table gives the mean and standard deviation and 95\% Confidence Interval(Cl). As can be seen, the mean value obtained for distance vision was less than the value of near of heterophoria. This value increased for near vision.

In this study, the results of the types about distance horizontal heterophoria showed that orthophoria just accounted for $7.3 \%$, while the other $92.7 \%$ ones had different kinds and extents of heterophoria (exophoria more than esophoria: $79.33 \%$ vs $13.33 \%$ ). and in the near heterophoria tests, $96.00 \%$ ones had different kinds and extents of heterophoria (exophoria:86.67\% vs esophoria: 9.33\%). Furthermore, the exophoria obtained for near vision was greater than that observed in distance vision (Table 2).

\section{Discussion}

Our investigation and statistical results indicated that the near exophoria was the most popular heterphoria in myopia juveniles. Sanker et al[5] found that 38 out of 49 college students were exophoria by near heterophoria tests. In 2007, Jin et al[6] tested 825 juveniles and found the percentage of esophoria, exophoria was $36.4 \%, 52.9 \%$ respectively in those students with normal refraction, and was $22.6 \%$ and $64.7 \%$ in the students with myopia. The distance exophoria, esophoria rate was $19.8 \%$ and $60.4 \%$ respectively in the students with normal refraction, while was $28.8 \%$ and $50 \%$ in the myopia students. Despite these studies had a discrepancy in their reported near and distance heterophoria tests, there was a pattern that near exophoria incidence was more than esophoria in the myopia population. Besides, some differences might exist in the distance and near heterophoria no matter in normal or myopia populations, which were consistent with our findings. We found the statistical difference $(F=300.00, \mathrm{P}=0.000)$ between the mean value of distance and near heterophoria . Meanwhile, the changes of convergence before and after glass wearing would affect heterphoria degree, leading to difference before and after visual correction. Therefore, we believed that heterophoria tests should include distance and near heterophoria before visual correction beyond of heterophoria after visual correction.

Heterphoria is believed to be associated with the development and progressive speed of myopia. Goss et al[7,8]found the association between near heterophoria and myopia. However, that association only occurred in those near heterophoria accompanying with accommodation lag [9]. In 2006, Adler et al[10] revealed that myopia increased faster in those near esophoria children than those orthophoria or exophoria ones, but with no significant difference $[11,12]$. Recently, myopia has become a social issue. In 2013, Fan et al[13]found that near exophoria juveniles accounted for $73.6 \%$ in those myopia ones, and these exophoria juveniles developed myopia faster than those esophoria and orthophoria ones, suggesting that the accommodation of eye position in juveniles is a risk factor of myopia, and 
reasonable optometry and accommodation practice might reduce the development of myopia. Therefore, each subject should have heterophoria tests to improve the comfort and success of optometry by excluding failure due to exophoria. Besides, practices could be administered according to the binocular vision test results to delay the myopia progress. The accurate measurement of heterophoria is important to myopia progress prediction and control selection.

There might be some differences of the heterphoria between normal and nearsighted populations. In 1980, Fang et al[14] investigated the heterophoria situations in 1156 normal visual acuity adolescents and the results showed that distance esophoria ones accounted for $44.63 \%$, and exophoria ones accounted for $24.48 \%$. In 2000, Ren et al[15] conducted heterophoria tests on 1027 navy pilots and found the ratio of orthophoria, distance esophoria, and exophoria was $13.1 \%, 48.4 \%$ and $23.8 \%$ respectively. In 1989, Xu et al[16] tested 650 eyes from 325 normal sighted subjects and they reported 295 cases of heterophoria (90.76\%), 30 cases of orthophoria(9.24\%). In those heterophoria ones, esophoria is the most common type (156 cases: $52.88 \%$ ). In 2003, Chen et al[17] performed heterophoria tests on 36 subjects including 11 myopia and 25 normal ones, their findings discovered about $72 \%$ normal sighted eyes had near exophoria, and $68 \%$ nearsighted eyes had near exophoria, but with no statistical significance. Despite the heterophoria degrees varied at those different studies, it's suggested that distance esophoria is more than exophoria, and near exophoria is more than esophoria in normal sighted healthy adolescents. As our research results disclosed, both distance and near exophoria was more than esophoria in nearsighted juveniles. The variance of our results from other publications might be due to the facts that included subjects were newly diagnosed myopia juveniles at their first optometry and they had no eyeglass wearing histories.

The approach of progressive multifocal eyeglass to control juvenile myopia is still in doubt, and most ophthalmologists and optometrists believe it should be selectively used. The progressive multifocal eyeglass wearing could add luminosity, decrease accommodation demand at near sight, enhance divergence, increase exophoria, reduce positive fusion, decrease accommodation, and increase accommodation lag. All these factors could increase myopia and therefore the progressive multifocal eyeglass should not be utilized in juveniles as routine. Furthermore, indications such as esophoria, high AC/A ratio should be strictly followed [18]. In 2012, a study showed that progressive multifocal eyeglass could control the development of myopia in those nearsighted children with high accommodation lag [19]. In 2011, Xu et al[20] reported their observations on 50 esophoria myopia subjects wearing eyeglass, and their results disclosed that the speed of degree increment was fastest in those subjects wearing normal eyeglass, then distance spectacles (-1.50DS) and multifocal eyeglass, and finally reading spectacles. The previous literature have reported [9,21,22]that bifocal or progressive multifocal eyeglass wearing could foster myopia in near exophoria children. Besides, most juveniles are not suitable to wear progressive eyeglass if they have normal visual functions. Although most myopia subjects wearing progressive multifocal eyeglass had no discomfort complaints, their optometry exams indicated a high percentage of exophoria and low AC/A ratio. Some subjects might loss convergence because of obvious intermittent exophoria problems. Progressive multifocal eyeglass might not cause serious outcomes in those juveniles with the normal position of the eye, however, it might turn to be serious in those ones with 
obvious exophoria without prism prescription. Only those juveniles having high AC/A ratio, esohoria, high positive relative accommodation (PRA) and negative relative accommodation(NRA) are suitable to wear progressive multifocal eyeglass. However, the juveniles meet above requirements account less than $20 \%$, therefore, optometrists should deliver their best prescription and suggestion at their abilities and systematically record AC/A ratio, position of the eye, amplitude of accommodation (AMP), PRA and NRA before prescribing progressive multifocal eyeglass.

Near visual fatigue has been paid more and more attention as the developing of society and the increasing burden of near distance visual assignments. In the case of the low heterphoria degree, it could be compensated itself with no symptom only by divergence reserve. When the heterphoria degree is large or there is convergence insufficiency, long term over the use of divergence reserve will lead to muscular visual fatigue. In 2016, Veselý et al[23] evaluated the binocular visions in 68 cases of young adults (mean age: 26 years), of whom 26 cases were both near and distance orthophoria, and 42 cases were abnormal (12 cases of esophoria, 8 cases of exophoria, 13 cases of convergence insufficiency). Their results suggested that accurate spherocylindrical lenses optical correction could alleviate binocular visual disorders. Therefore, different refractive error and deviation of eye position should be taken into consideration for optical correction so as to improve the relationship of accommodation and convergence and to reduce the deviation degree which would increase otherwise. It's necessary for optometrists to keep this knowledge in mind. In 2015, Vilela et al[24]reported visual fatigue accounted for $24.7 \%$ in 964 students aged between 6 to 16 years, reminding health care providers to pay attention to these visual fatigue students since the fatigue will affect those students' lives and studies in schools. At the time of refractive correction, we could ask the examinees to have optic correction or training for heterophoria according to their ages. In order to find tropia or heterophoria patients earlier, we believed that eye position and near as well as distance heterophoria should be measured during optometry, the optic correction prescription based on which could protect juvenile vision and visual function mostly.

\section{Conclusions}

In conclusion, this test reported the prevalence of heterophoria in west area of China. The prevalence of exophoria appears to be higher than esophoria in low myopia juvenile school students. Due to the high occurrence of heterophoria in myopia, it should be routinely assessed in clinical optometric practice.

\section{Abbreviations}

MPBVA: maximum plus for best visual acuity; Cl: confidence interval; D: diopter; SD: standard deviation; SE: spherical equivalent; DHT: the amount of distance horizontal heterophoria; NHT: The amount of near horizontal heterophoria.

\section{Declarations}

Acknowledgements 
Not applicable.

Funding

This study was supported by the National Nature Science Foundation of China (81341105); the Health Department of Sichuan Province (120442).

Availability of data and materials

The data have not been placed in any online data storage. The datasets generated and analyzed during the study are available upon request from the first author.

Authors' contributions

Study concept and design (ZYC); collection, management, analysis, and interpretation of data (YLY, LL, ZYC); and preparation, review, or approval of the manuscript (YLY, LL, WY, ZGP, ZYC). All authors read and approved the final manuscript.

YLY and LLcontributed equally to this work.

Ethics approval and consent to participate

This study followed the tenets of the Declaration of Helsinki and approved by the ethics committee of the Department of Optometry and Ophthalmology, North Sichuan Medical College. Informed written consent was obtained from the parents/legal guardians of all the participants.

Consent for publication

Not Applicable.

Competing interests

The authors declare that they have no competing interests

Author details

${ }^{1}$ Department of Optometry and Ophthalmology, North Sichuan Medical College, Nanchong, Sichuan province 637000 , China. ${ }^{2}$ Boshi Eye and E $\triangle N \otimes T \otimes H o s p i t a l \otimes N a n c h o n g \unrhd$ Sichuan

Province,637000,China. ${ }^{3}$ Department of Ophthalmology,the Suining Central Hospital,Suining, Sichuan Province,629000,China.

\section{References}

1. Cantó-Cerdán M, Cacho-Martínez P, García-Muñoz Á. Measuring the heterophoria: Agreement between two methods in non-presbyopic and presbyopic patients. Journal of Optometry. 2018 
,11(3):153-159.

2. Babinsky E, Sreenivasan V, Candy TR. Near Heterophoria in Early Childhood. Invest Ophthalmol Vis Sci.2015,56(2):1406-1415.

3. Lv F, Xu D, Qu J. A study on difference of ocular motor parameters in myopia. Chinese Journal of Ophthalmologic Medicine.2004;40(9):583-586.

4. 4.Scheiman M, Wick B. Clinical management of Binocular Vision. Heterophoric, Accommodative, and Eye Movement Disorders. 4th ed. Philadelphia: Lippincott Williams \& Wilkins; 2014.

5. Nijil Sanker囚Avinash Prabhu凶Avik Ray. A comparison of near-dissociated heterophoria tests in free space.Clin Exp Optom. 2012, 95(6): 638-642.

6. Jing HX, Shi GR, Guo XJ, Wu G.Horizontal phoria among myopic students and effects of ametropic correction on phoria. Inter J Ophthalm.2007,7(5):1470-1473.

7. Goss DA, Zhai H. Clinical and laboratory investigations of the relationship of accommodation and convergence function with refractive error. A literature review[J]. Doc Ophthalmol, 1994,86(4):349380.

8. Goss DA, Grosvenor T. Rates of childhood myopia progression with bifocals as a function of nearpoint phoria: consistency of three studies[J]. Optom Vis Sci.1990,67(8):637-640.

9. Gwiazda JE, Hyman L,Norton TT, Hussein M, Marsh-Tootle W, Manny R, et al. Accommodation and related risk factors associated with myopia progression and their interaction with treatment in COMET children. Invest Ophthalmol Vis Sci. 2004,45(7):2143-2151.

10. Daniel Adler冈Michel Millodot. The possible effect of undercorrection on myopic progression in children.Clin Exp Optom. 2006; 89(5): 315-321.

11. Yun LX, Li LG, Wu L,Chen XM,Zhang LN.Clinical study on factors affecting the development of juvenile myopia. Chinese Journal of Ophthalmologic Medicine.2016, 6(4):161-166.

12. Goss DA, Jackson TW.Clinical findings before the onset of myopia in youth: 3.heterophoria[J].Optom Vis Sci.1996, 73:269-278.

13. Fan SY, Zhang QS, Mu SS, Wang L.Risk factors of the development of juvenile myopia. Journal of Clinical Ophthalmology, 2013,21(5):447-450.

14. Fang LL, Yu JS, Zhang FH. Phoria and collection test in normal youth. Chinese Journal of Ophthalmologic Medicine.1980,16:153-157.

15. Ren XM, Wang Q. Phoria investigation on navy pilots. Journal of Navy Medicine.2000,2 1(1):50-51.

16. Xu GX,Cui SY. The study on status of ametropia, phoria and depth perception in 60 eyes with normal vision. Chinese Journal of Practical Ophthalmology1989,7(9):575-576.

17. ChenAH, Dom AAM.Heterophoria in young adults with emmetropia and myopia. Malaysian Journal of Medical Sciences. 2003,10(1):90-94.

18. He GH, Zhang YL, Zhang M. The side effect of progressive addition lense optometry on juvenile visual functions. Chinese Journal of Strabismus \& Pediatric Ophthalmology.2014,22(4): 38-39. 
19. David A. Berntsen,Loraine T. Sinnott,Donald O. Mutti,et al. A Randomized Trial Using Progressive Addition Lenses to Evaluate Theories of Myopia Progression in Children with a High Lag of Accommodation. Invest Ophthalmol Vis Sci. 2012, 53(2):640-649.

20. Xu Y, Zhao W, Cui ZL. A follow-up of esophoria in 50 myopes wearing spectacles for 4 months to 5 years. Inter J Ophthalm. 2011,11(1):73-75.

21. Goss DA.Effect of spectacle correction on the progression of myopia in children--a literature review. $J$ Am Optom Assoc.1994,65(2):117-128.

22. Fulk GW, Cyert LA. Can bifocals slow myopia progression? [J].J Am Optom Assoc.1996,67(12):749754.

23. Veselý P, Spurná G, Hanák L, Synek S.Disorders of Simple Binocular Vision in Heterophoria and their Spectacle Correction. Cesk Slov Oftalmol.2016,72(6):223-225.

24. Manuel AP Vilela, Victor D Castagno, rodrigo D Meucci. Anaclaudia G Fassa. asthenopia in schoolchildren. Clinical Ophthalmology. 2015,9:1595-1603.

\section{Tables}

\begin{tabular}{|c|c|c|c|}
\hline & Mean & SD & $95 \% \mathrm{CI}$ \\
\hline Age(years) & 13.71 & 1.25 & $13.51 \square 13.91$ \\
\hline $\mathrm{SE}(\mathrm{D})$ & -1.71 & 0.68 & $-1.82 \square-1.60$ \\
\hline $\mathrm{DHT}(\triangle)$ & -1.97 & 2.37 & $-2.3 \square-1.59$ \\
\hline $\operatorname{NHT}(\triangle)$ & -3.91 & 2.96 & $-4.38 \square-3.43$ \\
\hline
\end{tabular}

Abbreviations: CI, confidence interval; D, diopter; SD, standard deviation; SE, spherical equivalent; DHT, the amount of distance horizontal heterophoria; NHT, The amount of near horizontal heterophoria

Table 2 - The results of near and distance exophoria and esophora examinations in 150 myopia middle school students

\begin{tabular}{ccc}
\hline & Distant & Near \\
\hline Orthophoria (\%) & $11(7.3 \%)$ & $6 \square 4 \% \square$ \\
Exophoria (\%) & $119 \square 79.33 \% \square$ & $130 \square 86.67 \% \square$ \\
Esophoria (\%) & $20 \square 13.33 \% \square$ & $14 \llbracket 9.33 \% \square$ \\
Comparison of the near and distant heterphoria & $F=184.33 \square P=0.000$ \\
\hline
\end{tabular}


Page 10/10 